\title{
REFLUJO VESICOURETERAL BILATERAL GRAVE Y NEFROPATÍA RENAL EN PACIENTES VARONES RECIÉN NACIDOS: SÍNDROME DE VÁLVULAS-LIKE O MICCIÓN NO COORDINADA EN EL FETO VARÓN
}

\author{
Rafael Luque Mialdea y Rosa Martín-Crespo.
}

Urología Pediátrica. Servicio de Cirugía Pediátrica. Complejo Hospitalario de Toledo. Toledo. España.

\begin{abstract}
Resumen.- Diversos trabajos se han publicado en la última década, que diferencian el reflujo vesico ureteral (RVU) neonatal con afectación grave renal, de otro grupo mayoritario de neonatos con RVU neonatal sin lesión renal y los diagnosticados en edades más tardías generalmente relacionados con infección urinaria (ITU). Conviniendo en que el RVU forma parte de un amplio espectro tanto en la expresión clínica como por lo tanto de su patogénesis, se han descrito diversas teorías para explicar la existencia de este tipo de RVU congénito en varones. La existencia de una malfunción vesico uretral fetal, expresada postnatalmente con una vejiga de alto riesgo, definida ésta mediante estudio urodinámico en el 1 - trimestre de vida extrauterina, explica la aparición de RVU grave fetal con
\end{abstract}

afectación funcional de una o ambas unidades renales ya en el recién nacido y que precisa de su reconocimiento para un adecuado planteamiento terapéutico, éste cuadro lo hemos caracterizado como Síndrome de Válvulas-like o Micción no coordinada fetal en el varón.

Palabras clave: Reflujo neonatal. Disfunción vesical. Disfunción vesico uretral. Reflujo vesicoureteral fetal. Síndrome de válvulas uretra posterior like. Micción no coordinada.

Summary.- OBJECTIVES: Various papers differentiating neonatal vesicoureteral reflux (VUR) with severe renal damage from other predominant group of newborns with neonatal VUR without renal lesions and those diagnosed in older ages, generally in relation with urinary tract infection (UTI), have been published over the last decade. From the standpoint that VUR is part of a broad spectrum both in clinical expression as in pathogenesis, with different theories described to explain the existence of this type of congenital VUR in males. The existence of a fetal vesicourethral dysfunction, presenting after birth as a high risk bladder, which is defined by urodynamic tests in the first trimester, explains the appearance of severe fetal VUR with functional deterioration of one or both renal units at the time of birth; this entity must be diagnosed to establish the adequate therapeutic management. This clinical picture is named Valve like syndrome or male uncoordinated fetal voiding.

Keywords: Neonatal reflux. Bladder dysfunction. Vesicourethral dysfunction. Fetal vesicoureteral reflux. Posterior urethra Valve like syndrome. Uncoordinated voiding. 


\section{INTRODUCCIÓN}

La existencia de reflujo vesicoureteral (RVU) bilateral de alto grado en recién nacidos, principalmente varones, diagnosticados prenatalmente de uropatía grave con afectación renal funcional severa neonatal y sin existencia de factores coadyuvantes de lesión renal, infección urinaria, plantean debates entre los urólogos pediátricos sobre su etiología y el planteamiento terapéutico idóneo (1-5).

Diversos trabajos se han publicado en la última década, que diferencian el RVU neonatal con afectación grave renal, de otro grupo mayoritario de neonatos con RVU neonatal sin lesión renal y los diagnosticados en edades mas tardías y generalmente relacionados con ITU (2-6). Conviniendo en que el RVU forma parte de un espectro amplio de expresión clínica y por lo tanto de su patogénesis, se han descrito diversas teorías para explicar la existencia de este tipo de RVU en varones (5,7-10). Describimos hace 4 años, los 4 primeros casos de nuestra serie actual, que denominamos "Síndrome de válvulas like" (SVL) ○ S. de micción no coordinada en el feto varón" (5). por dos razones fundamentales, una diferenciar a éste grupo de neonatos -SVL- con el resto de neonatos, varones y mujeres, con RVU masivo sin lesión renal (2), y correlacionarlos patogénicamente con los neonatos portadores de válvulas de uretra posterior (VUP), dada la similitud de presentación - clínica y sintomática-, evolución y génesis diferente del denominador común, la obstrucción uretral, que en los pacientes de SVL sería de origen funcional (5), mientras que en los VUP sería de origen orgánico.

Presentamos 7 varones neonatos con diagnóstico de SVL y realizamos la caracterización del cuadro tras los estudios de imagen- ecografía prenatal, postnatal, cistografía permiccional y gammagrafía renal por DMSA. Durante el primer trimestre de vida en los pacientes de nuestra serie, se realizaron estudios urodinámicos para el despistaje de vejiga de alto riesgo (1 1-12), determinando la capacidad vesical funcional (CVF), presión de escape en reposo (PER), compliance vesical y residuo postmiccional.

\section{MATERIAL Y MÉTODOS}

Desde 1998 hemos tratado 34 neonatos varones, con diagnóstico prenatal de dilatación grave del tracto urinario superior, con reflujo vesico-ureteral (RVU) del recién nacido. Nuestro algoritmo diagnóstico de dilatación prenatal del tracto urinario superior (Figura 1), fue realizado en todos los pacientes (Tabla I).

De los 34 neonatos varones, 7 cumplen la caracterización del cuadro del S. de Válvulas Like (cuadro 1, MartínCrespo y cols., 2003) (5). En los 7 neonatos, el estudio ecográfico neonatal mostró una vejiga de paredes engrosadas ( > de $5 \mathrm{~mm}$ ), residuo elevado, ausencia de válvula y signos ecográficos renales de displasia-nefropatía por reflujo en 11 UR y megauréter en todas las UR. La cistografía permiccional seriada (CUMS) (Figura2), demostró la existencia de reflujo vesicoureteral masivo bilateral (14 UR) y en 6 de los 7 neonatos se comprobó una impronta a nivel del esfínter externo con ausencia de válvula uretral y de dilatación de la uretra prostática. El grado de afectación funcional renal presentó un amplio espectro, tanto analítica como por estudio de gammagrafia renal por DMSA. (Tabla II).

De los 7 pacientes de nuestra serie, 3 presentaron un diagnóstico prenatal y 4 postnatal precoz. Todos los pacientes de diagnóstico prenatal fueron inicialmente etiquetados de válvulas de uretra posterior, pero con unas diferencias significativas, no alteraron el volumen del lí-

\section{Diagnóstico prenatal de hidronefrosis Confirmación postnatal de hidronefrosis: Ecografia}

Ectasia

pelvis $</=1 \mathrm{~cm}$

Hidronefrosis pelvis

$>1<2.5 \mathrm{~cm}$ hidronefrosis pelvis $>/=2.5 \mathrm{~cm}$

\section{Control seriado ecográfico}
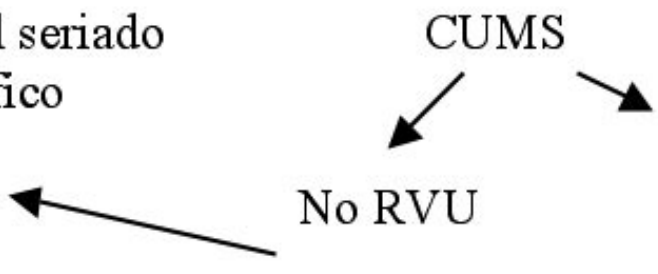

No RVU

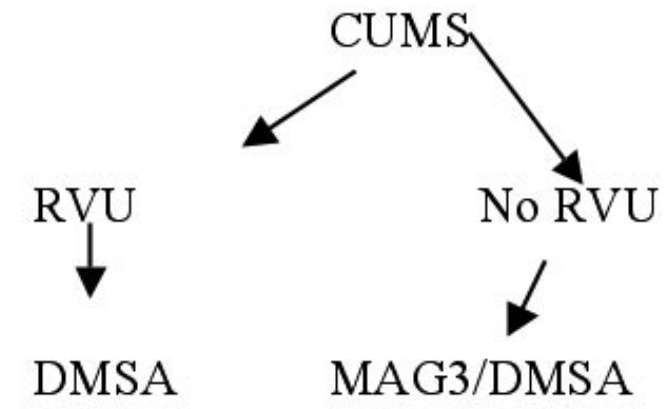

FIGURA 1. Protocolo de estudio de la hidronefrosis de diagnóstico prenatal. 


\section{TABLA I. LADO Y GRADO DE AFECTACIÓN DEL REFLUJO VÉSICO-URETERAL EN LOS 34 NEONATOS (N)} VARONES ESTUDIADOS I42 UNIDADES RENALES REFLUYENTES -UR-I.

\begin{tabular}{|l|c|c|c|c|}
\hline Lado & N & UR & \multicolumn{2}{|c|}{$\begin{array}{c}\text { Reflujo vesico-ureteral } \\
\text { Alto grado IV-V Bajo grado I-III }\end{array}$} \\
\hline Bilateral & 18 & 26 & 17 & 9 \\
\hline Unilateral & 16 & 16 & 9 & 16 \\
\hline Total & 34 & 42 & 26 & 7 \\
\hline
\end{tabular}

quido amniótico, fueron partos a término y no tuvieron pérdida del "bienestar fetal"; signos y síntomas todos ellos alterados en los casos de VUP y motivos de inducción de parto prematuro.

Nuestro planteamiento terapéutico fue similar al realizado en los casos de válvulas de uretra posterior, implantando como objetivo prioritario el de conseguir un sistema urinario de baja presión: descompresión urinaria mediante sondaje vesical con sonda de silicona, y en función a la respuesta de la creatinemia y disminución ecográfica de la dilatación del tracto urinario superior, realizar una derivación cutánea, que se mantuvo un periodo medio de 12 meses (entre 8-16 meses); las derivaciones realizadas han sido ureteropielostomía $(n=4)$ y vesicostomías $(n=5)$. El estudio urodinámico realizado en el 10 trimestre de vida, mostró la existencia de un tracto urinario de alta presión con hiperactividad del detrusor, baja acomodación (complíance media de $0.4 \mathrm{ml} / \mathrm{cm}$ de $\mathrm{H}_{2} \mathrm{O}$ ) y CVF disminuida, con una presión media de escape en reposo de $75 \mathrm{~cm}$

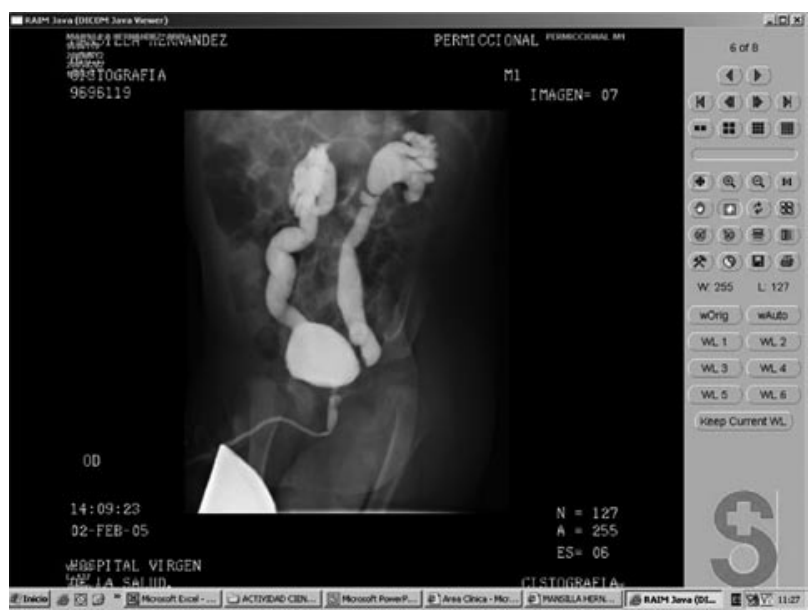

FIGURA 2. CUMS permiccional que muestra reflujo vesicoureteral bilateral de alto grado, bálonamiento de pelvis, megaúreter bilateral e impronta a nivel del esfínter externo en región periuretral. Ausencia de válvulas uretrales posteriores. de $\mathrm{H}_{2} \mathrm{O}\left(68-90 \mathrm{~cm}\right.$ de $\left.\mathrm{H}_{2} \mathrm{O}\right)$ y con residuo postmiccional elevado (>20\% de la CVF) (Figura3).

Al $3^{\circ}$ mes de la derivación se inició tratamiento medicamentoso con anticolinérgicos (cloruro de oxibutinina, a dosis de $0.2 \mathrm{mgrs} / \mathrm{Kg}$ de peso), manteniendo un tratamiento antibiótico profiláctico desde el nacimiento. Previo al cierre de la vesicostomía se coloca tapón de gastrostomía en el estoma de la derivación, y con él implantado y tras un periodo de 1 a 3 meses, se realizan los estudios valorativos de mejoría de estabilidad vesicoureteral La estabilización de la vía urinaria se considera adecuada tras estudio de ecografía, disminución del grosor de la pared vesical y disminución de la dilatación del tracto urinario superior, estudio de CUMS: disminución o desaparición del reflujo vesicoureteral y en estudio urodinámico mejoría o normalización de la CVF, PER. La cateterización vesical intermitente $(\mathrm{CVI})$ se instaura una vez cerrada la derivación urinaria para mantener un residuo bajo postmiccional.

Tras este planteamiento se realizó en 1 paciente nefrectomía izquierda con ureterocistoplastia y reimplantación ureteral mediante técnica de Politano-Leabetter, posteriormente precisó el mismo paciente de pielostomía cutánea derecha, que permanece en la actualidad. Únicamente en 2 pacientes se realizó como único tratamiento quirúrgico el cierre de la vesicostomía, sin precisar ulterior corrección quirúrgica del RVU. Por el contrario, en 4 pacientes tras cierre de la derivación urinaria fue necesario realizar corrección quirúrgica del reflujo vesicoureteral: en 2 casos mediante cirugía convencional: reimplantación ureteral tipo Cohen en 4 UR; y en 2 casos cirugía endoscópica, mediante la inyección submeatal de Deflux $®$ en 4 UR.

\section{RESULTADOS}

El seguimiento medio ha sido 3.8 años (1-8 años).

Función renal: La función renal es normal en la actualidad en todos los pacientes. Un paciente, el mayor de la serie, presenta un riñón único con función renal en cuanto a creatinemia por debajo de $0.9 \mathrm{mg} / \mathrm{dl}$, pero el riñón se encuentra derivado mediante una pielostomía por fracaso 
TABLA II. RESULTADOS DEL ESTUDIO CON DMSA EN LAS 42 UNIDADES RENALES REFLUYENTES (UR). CON ASTERISCO ${ }^{*}$ ), LAS 14 UR PERTENECIENTES A LOS 7 NEONATOS VARONES CON EL «SIINDROME DE MICCIÓN NO COORDINADA FETAL EN EL VARÓN»

\begin{tabular}{|l|c|c|c|c|c|}
\hline & \multicolumn{2}{|c|}{ RVU bilateral } & \multicolumn{2}{c|}{ RVU unilateral } & Total \\
\hline DMSA (FRD) & Alto grado 21 UR & Bajo grado 9 UR & Alto grado 6 UR & Bajo grado 6 UR & 42UR \\
\hline FRDUR & UR & UR & & & \\
\hline$<10 \%$ & $1^{*}$ & - & - & - & 1 \\
\hline $10-20 \%$ & $2^{*}$ & - & - & - & 2 \\
\hline $20-40 \%$ & $8^{*}$ & $3^{*}$ & 6 & - & 17 \\
\hline$>40 \%$ & 10 & 6 & - & 6 & 22 \\
\hline TOTAL & 21 & 9 & 6 & 6 & 42 \\
\hline
\end{tabular}

Reflujo vésico-ureteral; FRD: Función renal diferencial.

funcional del tracto urinario superior y secundariamente insuficiencia renal.

Reflujo vesicoureteral: El RVU desapareció tras corrección quirúrgica en 5 casos y en 2 casos con tratamiento anticolinérgico, tras el cierre de la vesicostomía, no recidivó. En 1 paciente, al año de la intervención del reflujo mediante cirugía endoscópica y tras suspensión del tratamiento con anticolinérgicos, recidivó el RVU activo bilateral de alto grado con insuficiencia renal, que mejoró radicalmente tras reanudar el tratamiento con anticolinérgico oral, con normalización de la función renal y remisión completa del RVU.

Función vesical: El tratamiento combinado, derivación urinaria e instauración de anticolinérgicos orales precoces, favorece la consecución de dos hechos. disminuir el tiempo de la derivación urinaria y una precoz estabilización vesicouretral. El cierre de la derivación se realiza cuando se consigue estabilidad del tracto urinario detectado mediante disminución de la dilatación urinaria, adecuación de la CVF y disminución de la PER. El parámetro de la complíance vesical es el más difícil de normalizar, manteniéndose en cifras inferiores de la normalidad en 4 pacientes. Solamente 2 pacientes de la serie mantienen parámetros adecuados de CVF, complíance y bajo residuo sin precisar para ello del tratamiento de base: anticolinérgicos y cateterización vesical intermitente. En 4 casos, de 5 años y 15, 20 y 24 meses de edad, se mantiene tratamiento con anticolinérgicos orales y los más pequeños con CVI. El caso derivado permanece pendiente de estudio urológico para plantearnos la viabilidad de una desderivación urinaria.

Continencia urinaria: Tres pacientes son continentes en la actualidad, precisando uno de ellos de anticolinérgicos orales. Tres pacientes son de corta edad y se encuentran en la fase de iniciar su continencia urinaria. El paciente derivado no es valorable en la actualidad.

\section{DISCUSIÓN}

La aparición en neonatos de reflujo vesico ureteral masivo bilateral con lesión renal, con espectro de afectación variable, predominante en el varón y con una malfunción vesico uretral, detectable en el primer trimestre de la vida mediante estudio urodinámico, se puede encuadrar en un proceso distinto de los casos de neonatos con reflujo vesicoureteral sin lesión renal y diferente a su vez del grupo de reflujos aparecidos en etapas ulteriores pediátricas (5). La similitud de la patogénesis de este grupo y de los varones afectos de válvulas de uretra posterior (VUP) (13), nos hicieron describir hace 4 años el síndrome de válvulas like o síndrome de micción no coordinada fetal en el varón (Martín-Crespo y cols., 2003). Ambas entidades presentan una expresión clínico sintomática similar en la etapa neonatal, con la gran diferencia de la existencia de válvulas de uretra en los pacientes con VUP y su ausencia en el SVL demostrada tanto por los métodos diagnósticos de imagen, como por endoscopia. La existencia de una pared vesical engrosada - prenatal y postnatalmente- y funcionalmente expresada por vejiga de baja compliance, puede explicar que la génesis de este tipo de patología sea debida a una obstrucción del tracto urinario inferior, orgánica como son los de válvula de uretra posterior, anomalías fetales uretrales (7-8), quistes de las glándulas de Cowper (9) ० funcional por malfunción del complejo vesico uretral fetal y expresado postnatalmente por la aparición de una impronta a nivel del esfínter externo durante la micción y por una vejiga de alto riesgo en el estudio urodinámico $(5,11$-16). 
El S. de válvulas Like presenta una caracterización típica (cuadro 1) y puede ser sospechado ya en el periodo prenatal por su historia evolutiva durante los estudios seriados prenatales a partir del $2^{\circ} 3^{\circ}$ trimestre. Los pacientes afectos de válvula de uretra posterior u obstrucción orgánica uretral, tienen una mayor repercusión en el volumen de líquido amniótico y por ende mayor repercusión en el desarrollo de hipoplasia pulmonar. De nuestra serie, 3 de los 4 pacientes con diagnóstico prenatal de uropatía grave, durante sus controles seriados ecográficos prenatales, ésta no afectó al volumen de líquido amniótico, ni tampoco en el bienestar fetal, indicaciones ambas de inducción de parto en el caso de fetos con VUP. Al contrario y durante el mismo periodo de estudio, los casos de VUP que se diagnosticaron prenatalmente, si afectaron a estos dos parámetros e indicaron el protocolo de actuación en todos ellos. La repercusión inmediata en el recién nacido, fue que los pacientes con SVL, no presentaron ningún problema de adaptación respiratoria ni precisaron maduración pulmonar fetal, a diferencia de aquellos diagnosticados de VUP que si precisaron de maduración fetal previo al nacimiento prematuro. Como describíamos en anterior publicación (5), las diferencias mas relevantes entre SVL y VUP y del grupo de neonatos con reflujo vesico ureteral sin repercusión renal, se ponen de manifiesto en los estudios de imagen realizados en la etapa neonatal. Las imágenes ecográficas neonatales en el SVL y VUP son idénticas a excepción de la falta de visualización de valva uretral posterior en los pacientes de VUP, pero ambas cuadros ecográficos forman un grupo diferenciador con respecto al grupo de RVU neonatal bilateral o unilateral sin afectación vesical. En este último grupo la vejiga y uretra se muestran normales, y la afectación ecográfica renal es mucho menor en cuanto a diferenciación córtico medular y masa renal. La CUMS muestra en el grupo de SVL una vejiga engrosada,

HOSPITAL PROVINCIAL DE TOLEDO

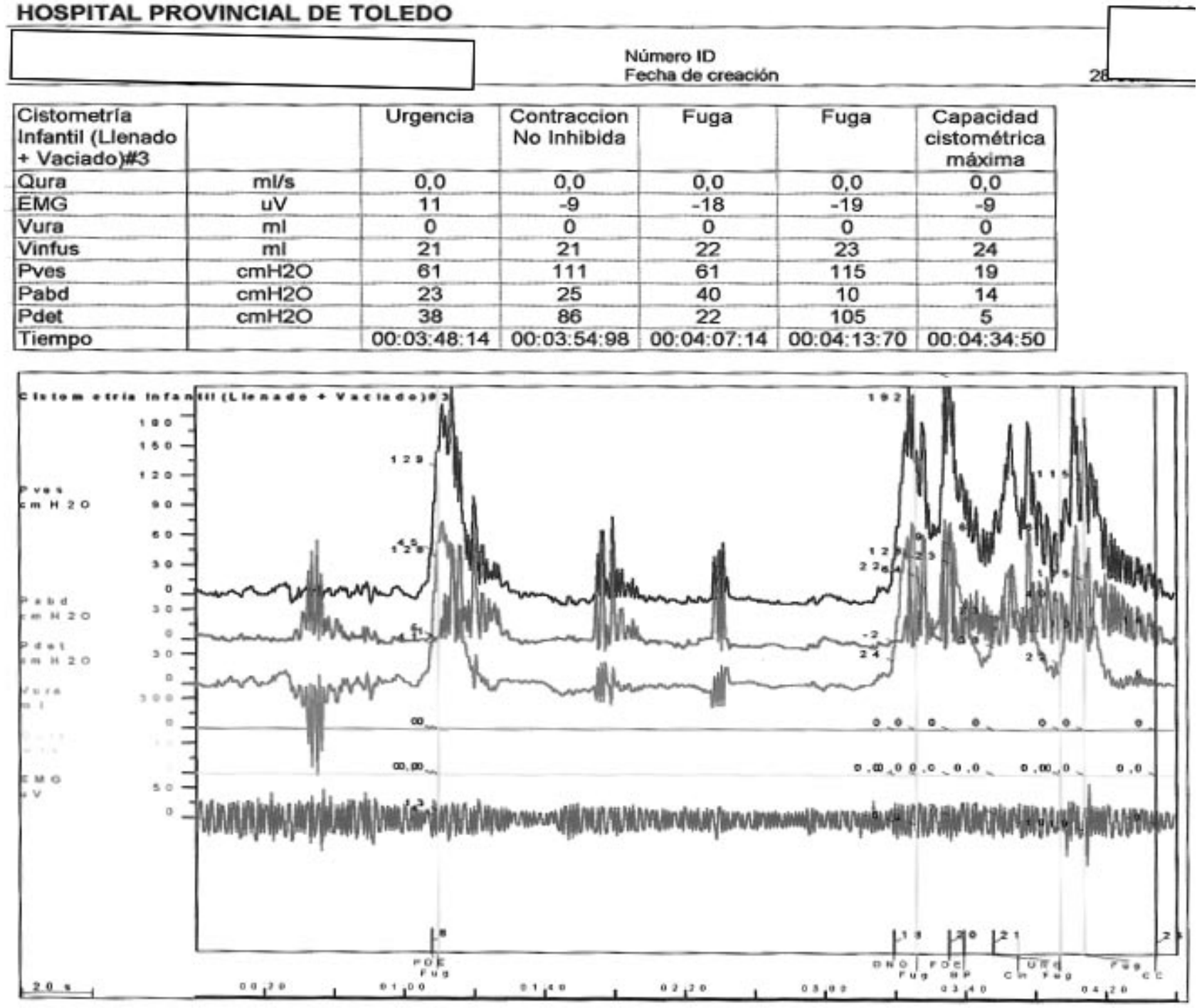

FIGURA 3. Estudio urodinámico de paciente con vejiga de alto riesgo por presentar S. Válvula-Like. Parámetros urodinámicos: Ritmo de infusión a $0.4 \mathrm{ml} / \mathrm{min}$. Resultados: Complíance final $0.1 \mathrm{ml} / \mathrm{cm}$ de $\mathrm{H}_{2} \mathrm{O}$ medida en el tramo final de la curva. Capacidad vesical máxima 24 con presión de $80 \mathrm{~cm}$ de $\mathrm{H}_{2} \mathrm{O}$. CVF de $15 \mathrm{ml}$ con PER de $120 \mathrm{~cm}$ de $\mathrm{H}_{2} \mathrm{O}$. Residuo postmiccional de $6 \mathrm{ml}$ el $20 \%$ de la CVM. 
trabecúlada y una impronta a nivel del esfínter externo en la serie miccional, con reflujo masivo y pelvis balonadas; en el grupo de VUP se describen los mismos hallazgos a excepción de presentar una uretra prostática dilatada y una imagen de valva uretral. El grupo de neonatos con RVU sin anomalía renal, la vejiga y uretra son normales aunque el reflujo puede presentar las mismas características que en los otros cuadros patológicos. En el estudio del DMSA en los portadores de SVL y VUP la afectación renal diferencial es la constante, mientras que en el grupo de RVU sin lesión renal, la función renal puede o no estar afectada, de hecho cuando en este grupo existe una lesión renal grave se revisa el paciente por si pudiera estar encuadrado en el grupo de SVL o bien ser portador de una valva uretral bulbar, de menor afectación en cuanto a expresión clínico sintomática en el periodo neonatal.

\section{Cuadro 1. Caracterización del cuadro Micción no coordinada o Síndrome de Válvulas Like} (Martín-Crespo y Luque Mialdea, 2003).

Neonatos varones (excepcionalmente mujeres)

\section{Diagnóstico prenatal: Ecografía}

- Diagnóstico prenatal antes de la 20 semana, generalmente 28-32 semana.

- Vejiga grande con pared gruesa y uropatía bilateral grave compatible con válvulas de uretra posterior.

- Dato diferencial intraútero: menor alteración del volumen de líquido amniótico.

\section{Recién nacido:}

Dato RN: No suele conllevar alteración funcional pulmonar.

Insuficiencia renal analítica en el $70 \%$

\section{Ecografía postnatal:}

Vejiga de pared gruesa (> 5mm).

Ausencia visualización de valva uretral, ausencia dilatación uretra prostática.

Ureterohidronefrosis bilateral.

Disminución masa renal, pobre diferenciación córtico -medular.

\section{Cistografía miccional seriada: (CUMS)}

- Imágenes en uretra:

- Impronta a nivel del esfínter uretral.

- Uretra calibre normal.

- Ausencia dilatación uretra prostática.

- Capacidad vesical variable.

- Reflujo vesicoureteral grados IV-V bilateral.

- Pelvis balonadas y dilatadas, kinking importante ureterales y pieloureteral.

\section{Estudio de gammagrafía renal (DMSA)}

Afectación bilateral (entre $20-40 \%$ de la función renal diferencial).

Nefropatía de reflujo ( $100 \%$ de los casos).

\section{Estudio Urodinámico (1ำ trimestre vida)}

Vejiga de alto riesgo: - baja compliance.

- baja capacidad vesical funcional (CVF).

- hiperpresión del detrusor.

- aumento actividad esfínteriana.

- elevado residuo.

Anatomía Patológica: Lesiones de fibrosis renal y focos de displasia renal.

\section{Evolución pronostica:}

- Insuficiencia renal crónica (28\%).

- Normalidad renal (72\%).

- Estabilización vesico uretral en el tiempo.

- Retardo de continencia en el tiempo: enuresis prolongada que guarda relación con micción no coordinada. 
En nuestros pacientes diagnosticados de SVL y VUP, se instaura estudio urodinámico sistemático durante el 1 trimestre de vida extrauterina; ambas patologías muestran una vejiga de alto riesgo $(5,11)$. En nuestra serie de SVL el estudio urodinámico realizado, diagnosticó en todos los casos la existencia de una vejiga de alto riesgo, la cual se caracteriza por una baja CVF, hiperpresión del detrusor, con presión media de escape en reposo de $75 \mathrm{~cm}$. de $\mathrm{H}_{2} \mathrm{O}$ y un elevado residuo miccional (>del $20 \%$ de la CVF) como expresión de una hiperactividad del esfínter. Estos datos urodinamicos son coincidentes con los descritos por otros autores (13-16) en estudios realizados en lactantes varones con RVU masivo y lesión renal severa. La malfunción vesicouretral expresada en los resultados urodinámicos, la determinan como una causa posible del reflujo $(5,11,14,16)$ o esta implicada en su cronificación (15).

Consideramos que este tipo de malfunción vesicouretral -micción no coordinada fetal- determina la patogénesis de una obstrucción uretral fetal, que en el caso del SVL es de carácter funcional. El planteamiento terapéutico a la vista de los estudios expuestos y sus resultados es similar a la realizada en aquellos otros neonatos con diagnóstico de VUP, ya que su principal diferencia terapéutica supone la necesidad de resección de la valva en los pacientes con VUP. El resto del planteamiento terapéutico es el mismo y su principal objetivo, también "conseguir un sistema urinario de baja presión". Para ello se realiza derivación cutánea en el periodo neonatal, y se mantiene durante el primer año de vida (8-10 meses de vida). Introducimos anticolinérgicos ora-

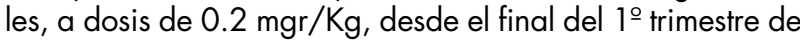
vida (12), con el objetivo de reducir el tiempo de derivación urinaria y obtener un sistema urinario de baja presión lo más precoz y duradero posible. El pronóstico evolutivo marca una clara diferencia entre este grupo de SVL con el de VUP y quizá de forma mas llamativa con el grupo de neonatos con RVU sin lesión renal severa. Este último grupo, se instaura un tratamiento médico, para nueva reevaluación a los 12-24 meses, debido a que el $60 \%$ de los mismos tiende a la desaparición del reflujo a estas edades $(2,6)$, por el contrario el pronóstico a largo plazo es diferente en los pacientes con SVL y/o VUP. Los pacientes portadores de VUP tienden a la cronificación en un porcentaje mayor que los pacientes de SVL (17), aunque éstos presentan según nuestra serie un $28 \%(n=2)$ de cronificación de la malfunción vesicouretral y son potencialmente candidatos a la insuficiencia renal terminal. No obstante el SVL presenta una tendencia autolimitada en su afectación y la adquisición de una adecuada estabilidad vesicoesfinteriana conlleva a la desaparición de la micción no coordinada, causante de su problemática. La adquisición de la continencia urinaria, micción completa sin residuo postmiccional son datos útiles de curación o mejoría del cuadro clínico y nos sirven para iniciar la interrupción del tratamiento de base. Las principales secuelas en nuestra serie han sido: nefropatía de reflujo en el $100 \%$ de los casos uni o bilateral aunque con función renal normal y retraso en la adquisición de la continencia urinaria.

\section{BIBLIOGRAFÍA y LECTURAS RECOMENDADAS ("lectura de interés $\mathrm{y}^{* *}$ lectura fundamental)}

**1. SCOTT, J.E.: "Fetal ureteric reflux: A follow-up study”. Br. J. Urol., 71: 481,1993.
**2. YEUNG, C.K.; GODLEY, M.L.; DHILLON, H.K. y cols.: "The characteristics of primary vesico-ureteric reflux in male and female infants with pre-natal hydronephrosis". Br. J. Urol., 80: 319, 1997.

**3. ARENA, F.; ROMEO, C.; CRUCCETI, A. y cols.: "Fetal vesicoureteral reflux: Neonatal findings and folowup study". Pedatr. Med. Chir., 23: 31, 2001.

*4. ARESES, R.; ARRUEBARRENA, D.; URBIETA, M.A. y cols.: "Severe primary vesicoureteral reflux in infants. A follw-up of 203 cases". Nefrología, 24: 131, 2004.

**5. MARTÍN-CRESPO, R.; LUQUE MIALDEA, R.: "Forma de presentación clínica del sídrome de miccion no coordinada en el varón " síndrome de válvulas like": Patrón urodinámico”. Cir. Pediatr., 16: 134, 2003.

*6. GODLEY, M.L.; DESAI, D.; YEUNG, C.K. y cols.: "The relationship between early renal status, and the resolution of vesico-ureteric reflux and bladder function al 16 moths"

**7. AVNI, E.F.; SCHULMAN, C.C.: "The origin of vesicoureteric reflux in male newborns: Further evidence in favour of a transient fetal urethral obstruction". Br. J. Urol., 78: 454, 1996.

*8. EBEL, K.D.: "Uroradiology in the fetus and newborn: Diagnosis and follow-up of congenital obstruction of the urinary tract". Pedatr. Radiol., 19: 247, 1989.

**9. DHILLON, H.K.; YEUNG, C.K.; DUFFY, P.G. y cols.: "Cowper's glands cysts: A cause of transient intra-uterine bladder outflow obstruction?". Fetal Diagn. Ther., 8: 51, 1993.

10. SHIMADA, K.; MATSUMOTO, F.; TOHDA, A. y cols.: "Histological study of fetal kidney with urethral obnstruction and vesicoureteral reflux: A consideration on the etiology of congenital reflux nephropathy". Int. J. Urol., 10: 518, 2003.

**11. McGUIRE, E.J.; WOODSIDE, J.R.; BORDEN, T.A.: "Upper urinary tract deterioration in patients with mtelodysplasia and detrusor hipertonia: A followup study". J. Urol., 129: 823, 1983.

12. LUQUE MILADEA, R.; MARTÍN-CRESPO, R.: "Uso del Cloruro de Oxibutinina en el Tratamiento de la Vejiga de Alto Riesgo en los niños menores de un año". Abstract Libro de Comunicaciones del XLII Congreso de la Sociedad Española de Cirugía Pediátrica, 28-31 Mayo, Valencia, 2003.

**13. REUTER, K.L.; LEBOWITZ, R.L.: "Massive vesicoureteral reflux mimicking posterior urethral valves in a fetus". JCU, 13: 584, 1985.

14. YEUNG, C.K.; GODLEY, M.L.; DHILLON, H.K. y cols.: "Urodynamic patterns in infants with normal lower urinary tracts or primary vesico-ureteric reflux". Br. J. Urol., 81: 461, 1998.

*15. PODESTA, M.L.; CASTERA, R.; RUARTE, A.C.: "Videourodynamic findings in young infants with severe primary reflux". J. Urol., 171: 829, 2004.

*16. CHANDRA, M.; MADDIX, H.: "Urodynamic dysfunction in infants with vesicouretral reflux". J. Pediatr., 136: 754, 2000.

17. LÓPEZ PEREIRA, P.; ESPINOSA, L.; MARTINEZ URRUTIA, M.J. y cols.: "Posterior urethral valves: Pronostic factors". BJU Internacional, 91: 687, 2003. 